\title{
Advanced glycation end products-cytokine-nitric oxide sequence pathway in the development of diabetic nephropathy: aminoguanidine ameliorates the overexpression of tumour necrosis factor- $\alpha$ and inducible nitric oxide synthase in diabetic rat glomeruli
}

\author{
H. Sugimoto ${ }^{1}$, K. Shikata ${ }^{1}$, J. Wada ${ }^{1}$, S. Horiuchi ${ }^{2}$, H. Makino ${ }^{1}$ \\ ${ }^{1}$ Department of Medicine III, Okayama University Medical School, Okayama, Japan \\ ${ }^{2}$ Department of Biochemistry, Kumamoto University Medical School, Kumamoto, Japan
}

\section{Abstract}

Aims/hypothesis. Advanced glycation end products are believed to contribute to diabetic microvascular complications by inducing glomerular damage but their role has not been fully clarified. In this study, we explain their central role in the induction of inducible nitric oxide synthase and production of nitric oxide (NO) in streptozotocin-induced diabetic rat glomeruli.

Methods. Localization of carboxymethyllysine, which is one of the chemical components of advanced glycation end products, glomerular expression of inducible nitric oxide synthase and urinary excretion and glomerular production of $\mathrm{NO}_{2}^{-} / \mathrm{NO}_{3}^{-}$were examined at 0 , 26,51 , and 52 weeks after the induction of diabetes. Therapeutic effects of aminoguanidine were also examined.

Results. Carboxymethyllysine was detected in the mesangial area in glomeruli and it progressively accumulated during 52 weeks of observation. Immunohis- tochemistry and hybridization studies in situ showed that the number of inducible nitric oxide synthasepositive cells was notably increased in diabetic rat glomeruli at 52 weeks. Further, this augmented expression paralleled intraglomerular expression of TNF- $\alpha$ and $\mathrm{NO}_{2}{ }^{-} / \mathrm{NO}_{3}{ }^{-}$in diabetic rat glomeruli. Treatment with aminoguanidine reduced the expression of TNF- $\alpha$, inducible nitric oxide synthase and intraglomerular $\mathrm{NO}_{2}{ }^{-} / \mathrm{NO}_{3}{ }^{-}$production. It also ameliorated proteinuria in diabetic rats.

Conclusion/interpretation. This study showed that carboxymethyllysine possibly enhances the expression of inducible nitric oxide synthase by stimulating the expression of TNF- $\alpha$ in diabetic rat glomeruli. The carboxymethyllysine-cytokine-NO sequence pathway could be one of the major mechanisms in the development of diabetic nephropathy. [Diabetologia (1999) 42: 878-886]

Keywords Advanced glycation end products, aminoguanidine, nitric oxide, diabetic nephropathy.
Received: 11 September 1998 and in revised form: 28 December 1998

Corresponding author: H. Sugimoto, MD, Department of Medicine III, Okayama University Medical School, 2-5-1 Shikata-cho, Okayama, 700, Japan

Abbreviations: AGEs, Advanced glycation end products; CML carboxymethyllysine; STZ, streptozotocin; NO, nitric oxide; NOS, nitric oxide synthase; $e$ NOS, endothelial cell nitric oxide synthase; $i$ NOS, inducible type nitric oxide synthase; $n$ NOS, neuronal type nitric oxide synthase; SD rats, Sprague Dawley rats.
It is widely considered that persistent hyperglycaemia is the primary causal factor in the development of diabetic nephropathy [1]. Advanced glycation end products (AGEs) are believed to be one of the major factors which contribute to chronic diabetic complications. We previously reported that carboxymethyllysine (CML), which is one of the chemical components of AGEs, accumulates in human and rat diabetic glomeruli $[2,3]$.

In recent studies, it has been shown that nitric oxide (NO), a labile soluble compound, is important in cell signalling, vasodilatation and cell-mediated immunity [4]. Nitric oxide is synthesized from L-arginine and molecular oxygen by the enzyme NO syn- 
Table 1. The number of rats killed each week

\begin{tabular}{lccccc}
\hline No of killed rats & $0 \mathrm{wk}$ & $26 \mathrm{wk}$ & $51 \mathrm{wk}$ & $52 \mathrm{wk}$ & Total \\
\hline Control & 5 & 5 & 5 & 5 & 20 \\
STZ & & 5 & 5 & 5 & 15 \\
STZ + AG & & 5 & & 5 & 10 \\
STZ + anti TNF & & & 5 & 5 & 10 \\
STZ + rabbit IgG & & & 5 & 5 & 10 \\
\hline
\end{tabular}

wk: week, AG: aminoguanidine, antiTNF: anti-TNF $\alpha$ antibody

thase (NOS), of which there are several distinct isoforms. Endothelium-derived NOS ( $e$ NOS) and neuronal NOS $(n \mathrm{NOS})$ are constitutive, $\mathrm{Ca}^{2+}$-dependent, agonist-triggered enzymes. The inducible type NOS $(i \mathrm{NOS})$ is induced at the transcriptional and translational levels by endotoxin or various cytokines or both. Induction of this isoform has been shown in a variety of cells and tissues such as macrophages [5], vascular smooth muscle and mesangial cells [6]. It possibly has important pathophysiological activities, by generating large amounts of NO with cytotoxic and cytostatic effects.

It has recently been shown that AGEs stimulate the expression of IL- 1 and TNF- $\alpha$ on macrophages and mesangial cells through binding to AGE receptors in vitro [7]. These cytokines are known to induce the expression of $i$ NOS on glomerular mesangial cells and macrophages [8]. These findings suggest it is pos- sible that AGEs induce the expression of $i$ NOS by enhancement of cytokine expression. In our study using streptozotocin (STZ)-induced diabetic rats, we verify the hypothesis that CML contributes to glomerular damage by enhancement of $i$ NOS expression through the overexpression of inflammatory cytokines in diabetic glomeruli. We examined the localization of CML and the expression of $i$ NOS and TNF- $\alpha$ in glomeruli using an immunohistochemical method. In addition, mRNA expression of $i$ NOS in glomeruli was examined by a nonradioactive in situ hybridization technique. Moreover, we explain the therapeutic effects of aminoguanidine on accumulation of CML and expression of $i$ NOS and TNF- $\alpha$ in glomeruli and on urinary protein excretion in STZinduced diabetic rats.

\section{Materials and methods}

Animals. The principle of laboratory animal care of the National Institute of Health (NIH) guideline was followed in all these experiments. Male Sprague Dawley (SD) rats were purchased from Charles River Japan (Yokohama, Japan). Male SD rats weighing about $120 \mathrm{~g}$ (4 weeks of age) were used in this study. These rats received a standard chow and water diet.

Induction of diabetes. Diabetes was induced in rats as described previously [9]. Five control rats (of total $n=20$ ) were killed at $0,26,51$ and 52 weeks after the injection with buffer.

Table 2. Changes in metabolic data after induction of diabetes

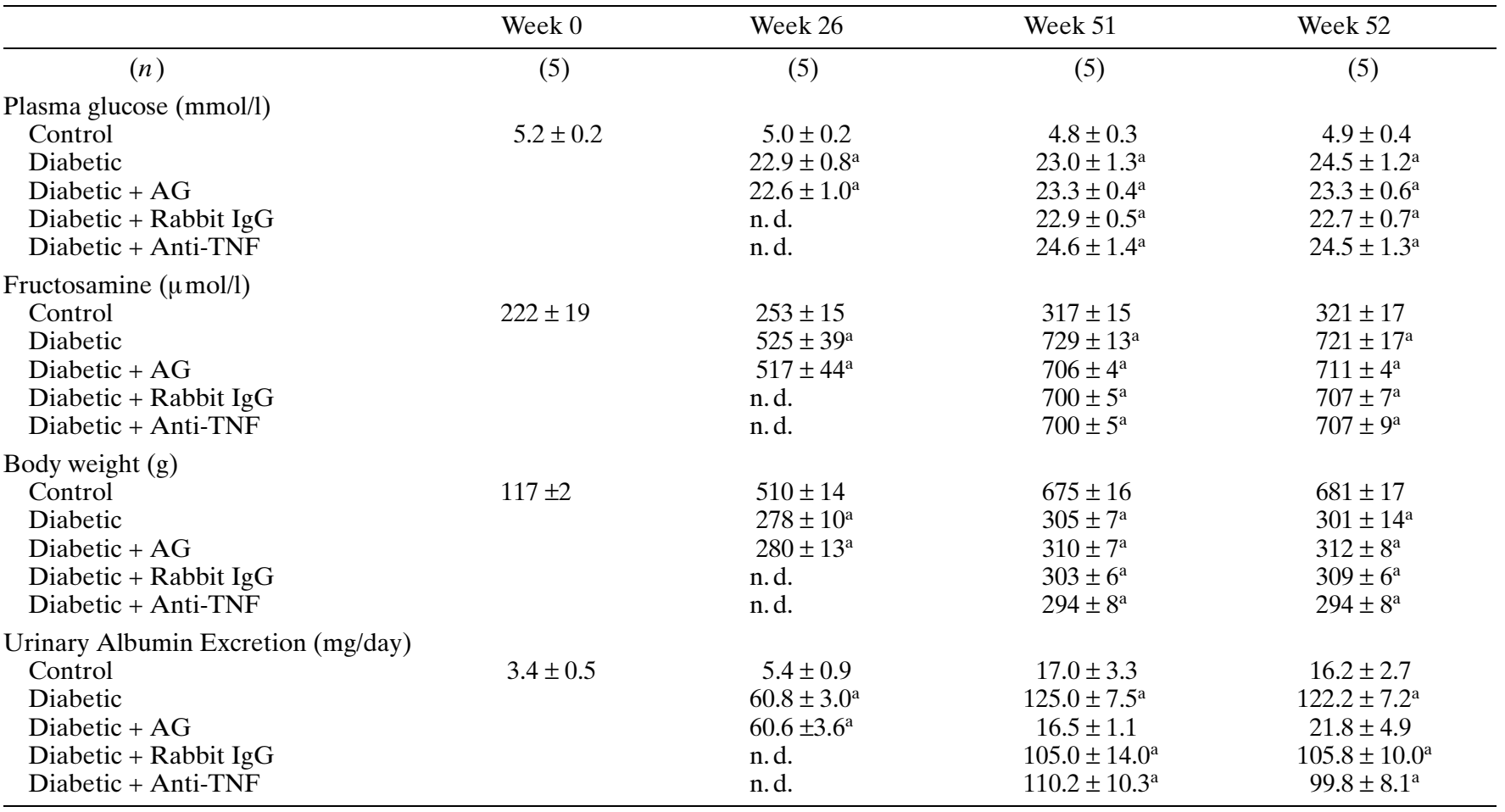

Data are means \pm SEM, n. d.: not done, $A G$ : aminoguanidine

${ }^{a} p<0.05$ vs control rats 


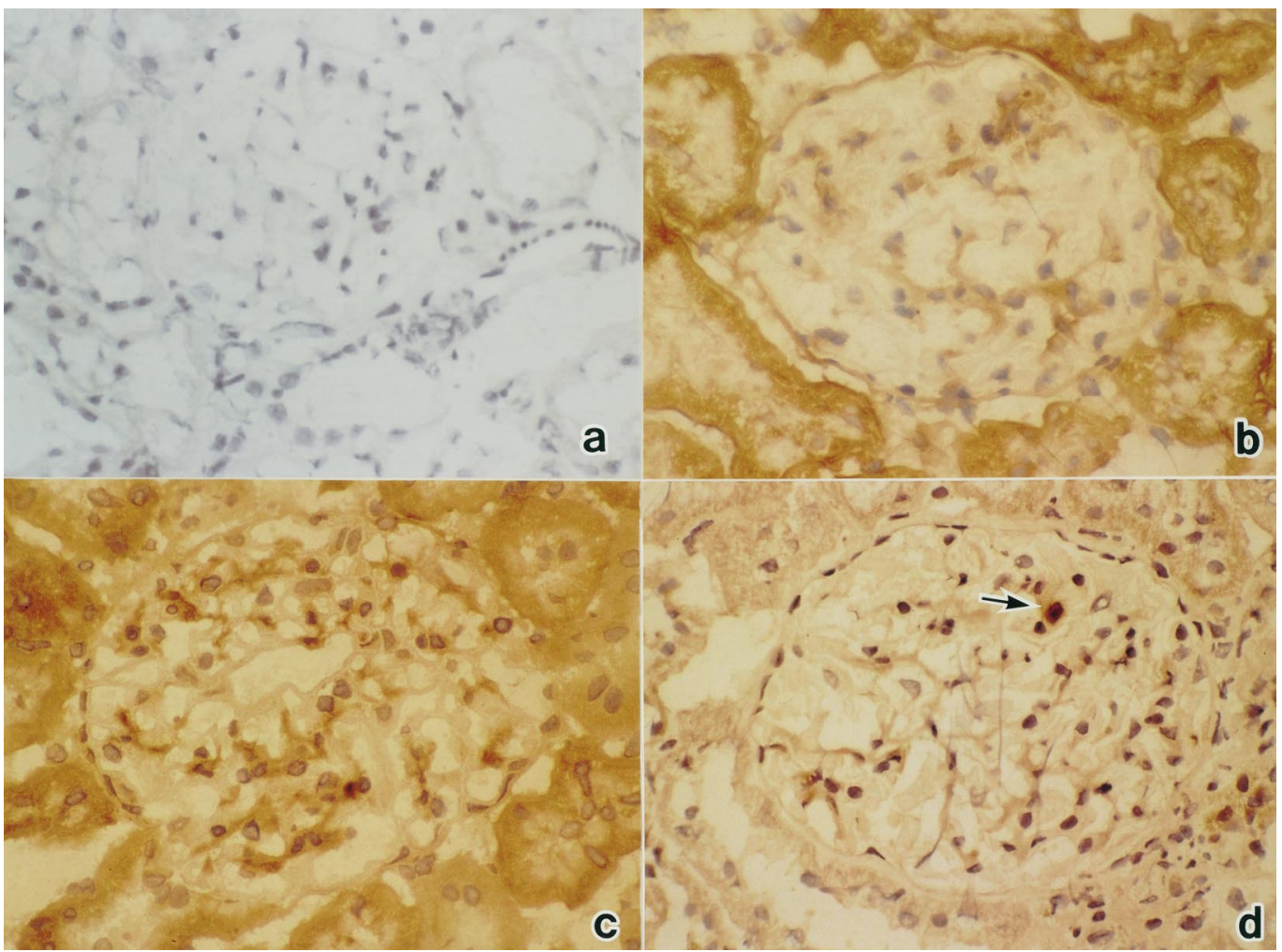

Fig. 1.a-d Immunoperoxidase staining for CML with monoclonal anti-CML antibody in normal-rats at 0 weeks (a) and 52 weeks (b) and in STZ-induced diabetic rats at 52 weeks (c) and in diabetic rats treated with aminoguanidine at 52 weeks (d). (c) Note strong staining in the mesangial regions. (d) Note some positive staining in the cytoplasm of the macrophage (arrows). Magnification: $\times 400$

Experimental rats were killed after injection with streptozotocin (STZ) or buffer and processed for immunohistochemical study. To study the therapeutic effects of aminoguanidine treatment, 26 weeks after the injection of STZ $(n=10)$, half $(n=5)$ of the diabetic rats were further selected at random to receive $1 \mathrm{~g} / \mathrm{l}$ aminoguanidine (Fluka Chemica, Buchs, Switzerland) in the drinking water. Since preliminary studies showed that $i$ NOS expression was not apparent at 26 weeks but was increased at 52 weeks, aminoguanidine was started at 26 weeks to test the therapeutic effects of aminoguanidine. All these rats were killed at 52 weeks and processed for immunohistochemical study. The number of rats killed each week are summarised in Table 1. Metabolic data were measured as described previously [9]. Urine samples were assayed for the stable $\mathrm{NO}$ metabolic end products, $\mathrm{NO}_{2}$ and $\mathrm{NO}_{3}$ with a nitrate/ nitrite assay kit (Cayman Chemical, Ann Arbor, Mich., USA) [10].

Effects of anti-TNF- $\alpha$ antibody. To study the effects of anti-rat TNF- $\alpha$ antibody (Innogenetics, Zwijndrecht, Belgium), 10 diabetic rats of 51 weeks after the injection of STZ $(n=10)$ were divided equally between a treatment group and a control group. From our previous (unpublished) data we know that $3 \mu \mathrm{g}$ of rat recombinant TNF $\left(1 \times 10^{7} \mathrm{U} / \mathrm{mg}\right)$ amplifies to develop proteinuria in rats with nephrotoxic nephritis. The treatment group were given $0.5 \mathrm{mg}$ of this antiserum, which neutralizes about $10 \mu \mathrm{g}$ of the TNF. The control group were given the equivalent dose of purified rabbit IgG (Jackson Immunoresearch Laboratories, West Grove, Pa., USA). The antibodies were injected subcutaneously every other day for 7 days at which point all rats were killed and processed for immunohistochemical study.

Antibodies. As primary antibodies, we used polyclonal antibodies (mAbs) against rat $i$ NOS (Calbiochem, San Diego, Calif., USA) and rat $e$ NOS (Transduction Laboratory, Ky., USA), biotinylated-anti-CML antibody (6D12) [11] and polyclonal antibody against rat TNF- $\alpha$ (Innogenetics, Zwijndrecht, Belgium). As secondary antibodies, biotinylated goat antimouse IgG and biotinylated goat anti-rabbit IgG, both of which lack cross-reaction with rat IgG, were obtained from Jackson Immunoresearch Laboratories (West Grove, Pa., USA).

Immunohistochemical procedure and scoring of immunostaining for $C M L, T N F$ - $\alpha$. Immunoreactivity of CML, TNF- $\alpha$ and $i$ NOS was studied with immunoperoxidase techniques by the labelled streptoavidin-biotin method [2]. We evaluated the scoring of immunostaining for CML and TNF- $\alpha$ according to a method described previously [12].

Quantification of iNOS-positive cells. Intraglomerular iNOSpositive cells were counted in 50 glomeruli per animal and expressed as the average number per glomerulus.

Histochemical staining procedure for NADH dehydrogenase. The catalytic activity of NOS was shown by enzymatic reduction of nitro blue tetrazolium (NBT) in the presence of NAD$\mathrm{PH}[11]$.

Oligonucleotide probe. Synthetic antisense and sense oligonucleotide probes (40 bases) of rat $i$ NOS were purchased from Takara Biochemicals (Osaka, Japan). According to the computer-based homology similarity search program (Soft Ware Developing, Toko, Japan) of all nucleic acid sequences in ro- 


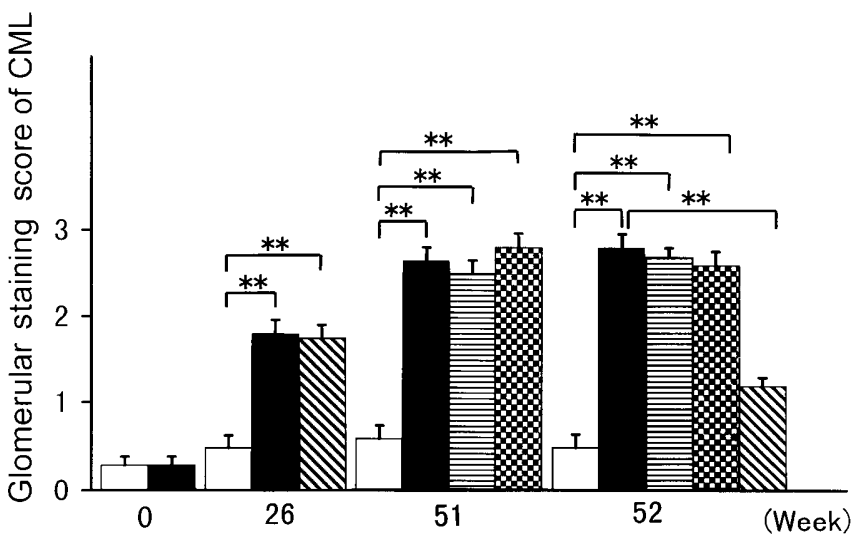

Fig. 2. Glomerular immunohistochemical staining scores for CML at 26, 51 and 52 weeks after STZ injection (closed bars) as compared with age-matched controls (open bars), aminoguanidine-treated diabetic rats (hatched bars), and anti-TNF$\alpha$ antibody-treated diabetic rats (latticed bars). $* * p<0.01$; $\square$ CONT, $\square$ STZ, $\mathbb{S} T Z+A G$, 圆STZ + rabbit IgG, $\mathrm{STZ}+$ Anti-TNF

dents stored in the GenBank, these oligomers have no homology with any other known nucleic acid sequences. Each probe was labelled with digoxigenin-labelled dUTP using a Dig Oligonucleotide Tailing Kit (Boeringer Mannheim Biochemica, Indianapolis, Ind., USA) according to the manufacturer's instructions.

In situ hybridization. In situ hybridization was done according to a previous report [9].

Glomerular $\mathrm{NO}_{2} / \mathrm{NO}_{3}$ production. Diabetic rats including those treated with aminoguanidine were anaesthetized with diethylether and glomeruli were isolated by mechanical sieving in sterile conditions and plated out in 4X6-well plates at a concentration of 7000 glomeruli per well. Glomeruli were incubated at $37^{\circ} \mathrm{C}$ in endotoxin-free RPMI 1640 culture medium (Gibco, Rockville, Md., USA) supplemented with $10 \%$ fetal calf serum. Nitrite production was determined in the supernatant for 24-h incubation with a nitrate/nitrite assay kit.

Statistical analysis. Values are expressed as means \pm SEM. The significance of the differences between two groups was analysed by Wilcoxon's test. Comparisons among three groups were done by two-way analysis of variance (ANOVA) followed by Scheffe's test to evaluate the significance of the differences between any two groups. A $p$ value of less than 0.05 was considered as statistically significant.

\section{Results}

Metabolic data. Body weight, blood glucose, fructosamine are shown in Table 2. All diabetic rats were moderately hyperglycaemic and had lower body weights. An increase in urinary protein in diabetic rats was noted at 26 and 52 weeks. Proteinuria was ameliorated by aminoguanidine at 52 weeks $(p<0.05)$.

Fig. 3.a-d Immunohistochemical localization of $i$ NOS in (a) a control rat kidney at 52 weeks, (b) STZ-induced diabetic rat kidney at 52 weeks, (c) an aminoguanidine-treated diabetic rat kidney at 52 weeks, (d) an anti-TNF- $\alpha$ antibody-treated diabetic rat kidney at 52 weeks. (d) Note that the $i$ NOS-positive cell (arrow) is morphologically macrophage. Magnification: $\times 400$

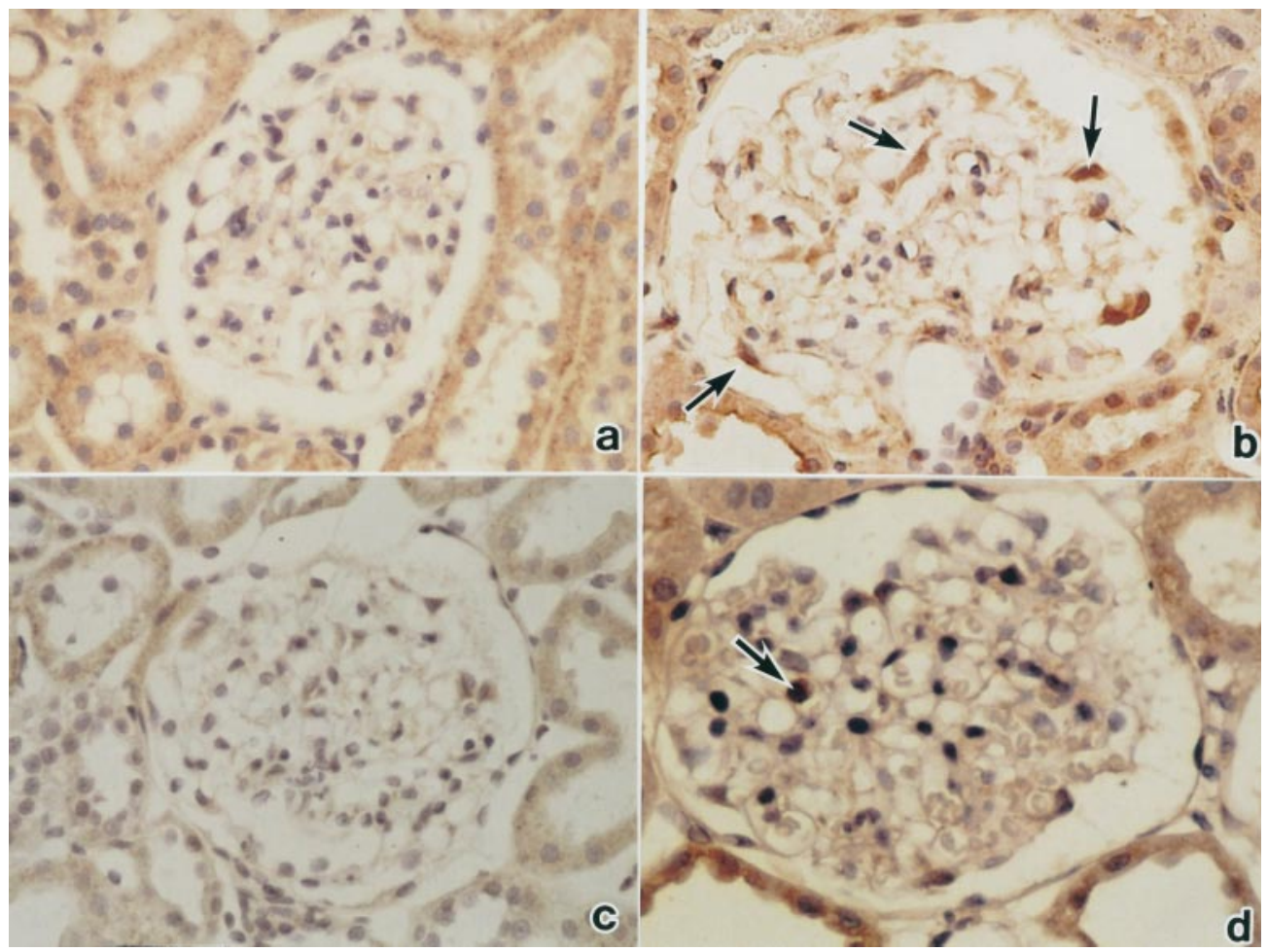




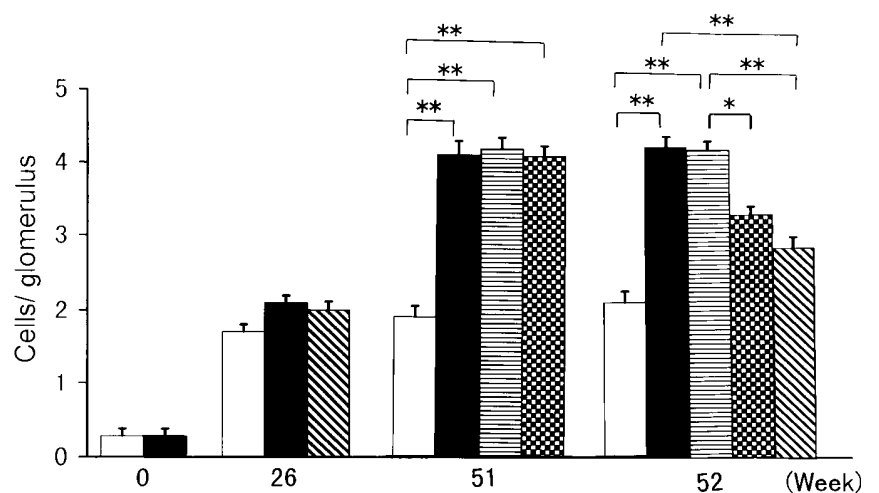

Fig.4. The number of $i$ NOS-positive cells in control rat glomeruli was gradually increased with ageing, which paralleled the CML staining. The number of $i$ NOS positive cells in glomeruli of STZ-induced diabetic rats was increased compared with that in control rat glomeruli at 52 weeks. Treatment of diabetic rats with aminoguanidine or anti-TNF- $\alpha$ antibody reduced the number of $i$ NOS-positive cells in glomeruli at 52 weeks. $* p<0.05, * * p<0.01 ; \square$ CONT, $\square \mathrm{STZ}, \mathbb{S} \mathrm{STZ}+$ $\mathrm{AG}, \mathrm{STZ}+$ rabbit IgG, STZ + Anti-TNF

Fig.5.a-d NADPH diaphorase staining in (a) a control rat kidney at 52 weeks, (b) a STZ-induced diabetic rat kidney at 52 weeks, (c) an aminoguanidine-treated diabetic rat kidney at 52 weeks, (d) anti-TNF- $\alpha$ antibody-treated diabetic rat kidney at 52 weeks. Magnification: $\times 200$. Arrows indicate NAD$\mathrm{PH}$ diaphorase activity. (d) Note that the NADPH diaphorase staining-positive cell (arrow head) is morphologically macrophage
CML staining. In normal rat glomeruli, CML staining was barely detected and was weak in the tubules at 0 weeks (Fig. 1 a). From 26 to 52 weeks, very faint staining was detected in the glomerular mesangium. The intensity of tubular staining progressively increased with time, i.e., ageing (Fig.1b). In STZ-induced diabetic rats glomerular staining was evident at 26 weeks and increased at 52 weeks. The glomerular staining score was higher in diabetic rats than age-matched control rats throughout the experimental period. (Fig. 2).

Staining for iNOS protein. There was no difference in the extraglomerular distribution of $i$ NOS protein by immunohistochemical staining between STZ diabetic and control rats from 0 to 52 weeks (Fig. 3). In control rats, there was only weak staining for $i$ NOS within the glomeruli throughout the experimental period (Figs. $3,4)$. The number of $i$ NOS-positive cells in control rat glomeruli gradually increased with ageing, which paralleled the CML staining. There were more $i$ NOS-positive cells in the glomeruli of STZ-induced diabetic rats than in those of the control rats at 52 weeks (Figs. $3,4)$. Treatment of diabetic rats with aminoguanidine or anti-TNF- $\alpha$ antibody reduced the number of $i$ NOS-positive cells in glomeruli at 52 weeks.

NADPH diaphorase activity. In the glomeruli of STZinduced diabetes, staining intensity of NADPH dia-

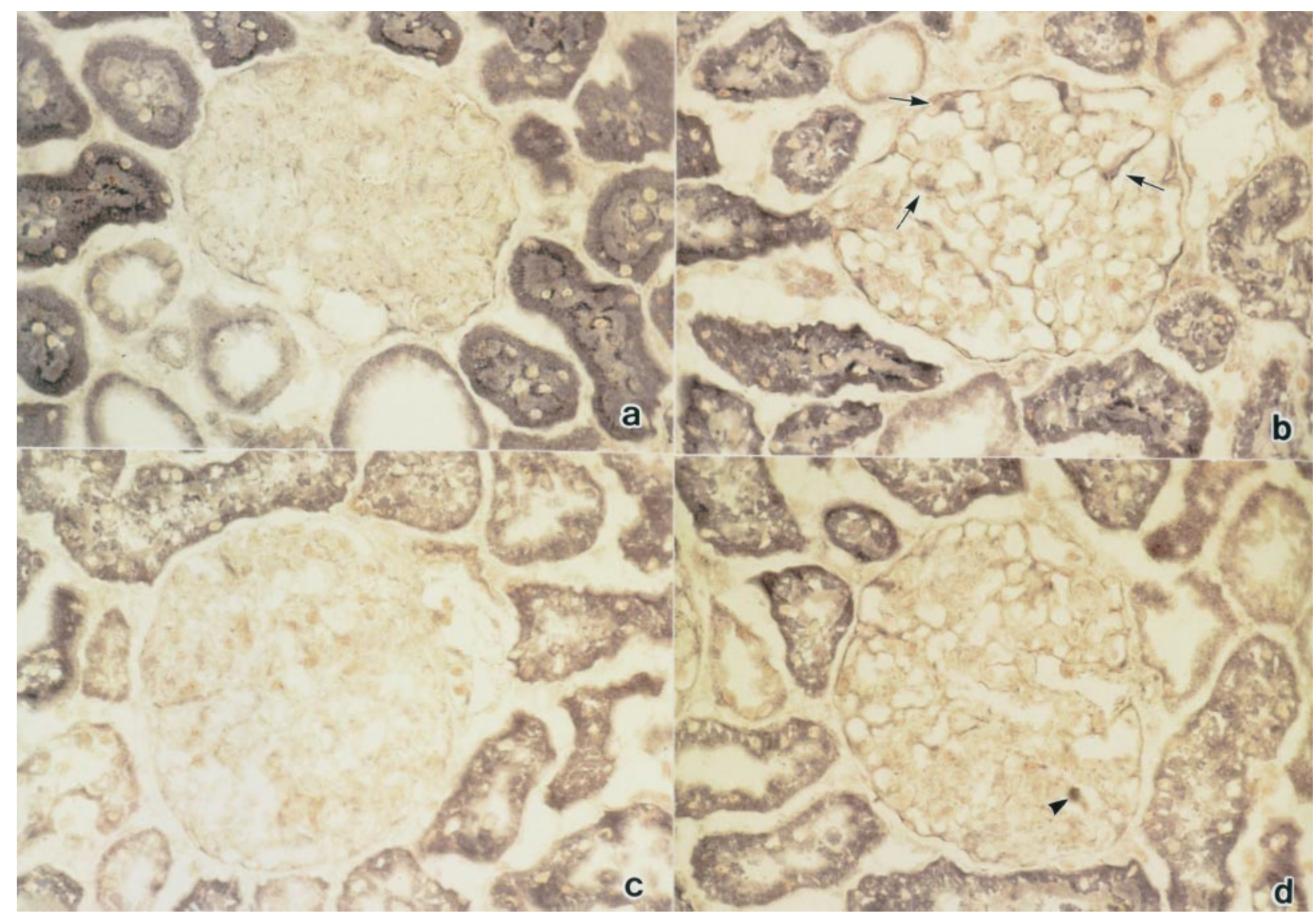




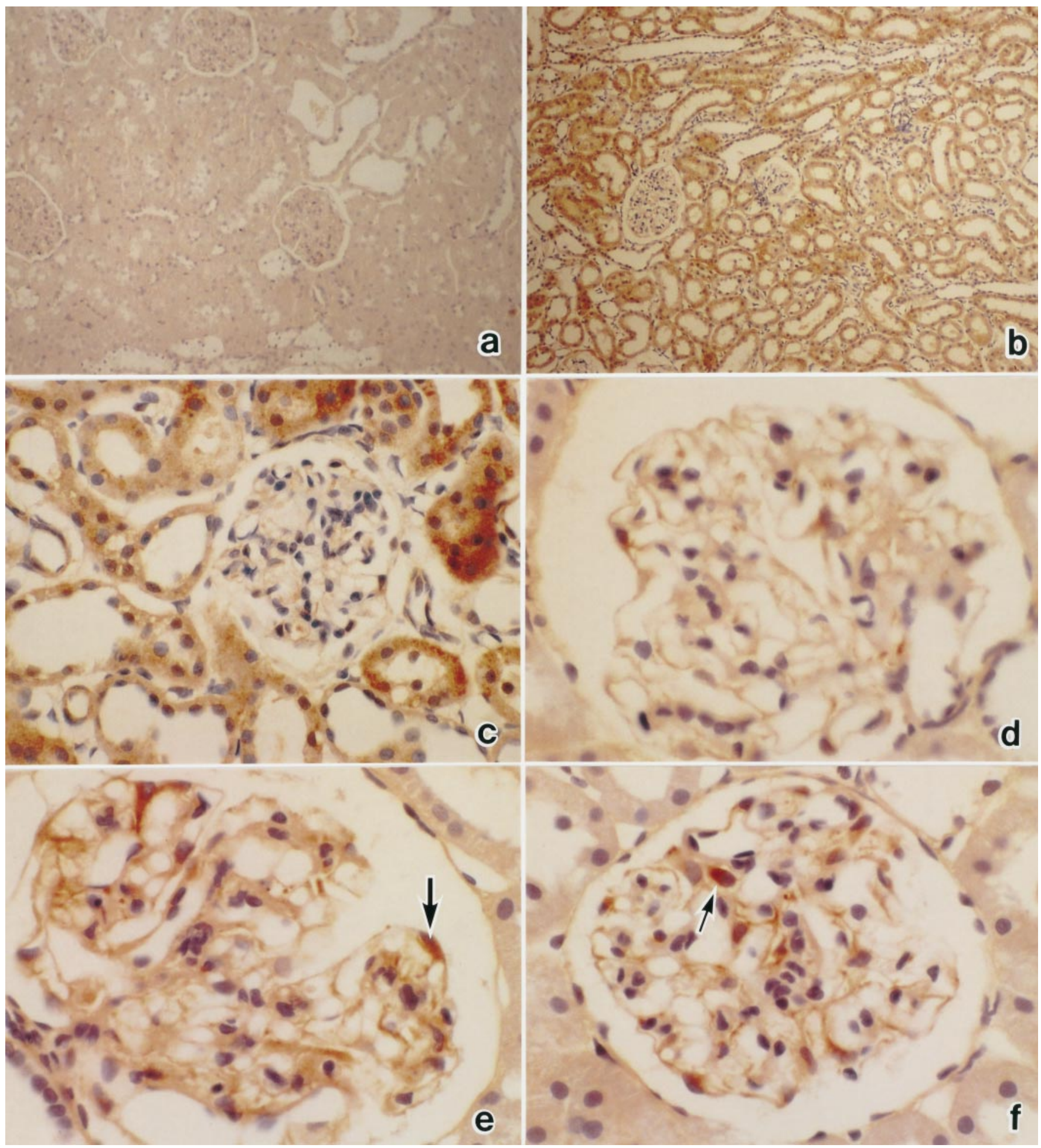

Fig. 6.a-f Localization of $i$ NOS mRNA (a) In STZ-induced diabetic rats at 52 weeks hybridization with the sense probe did not label any renal structures. (b) Hybridization with antisense probe yielded positive labelling of cortical thick ascending limb (TAL), proximal tubules, collecting ducts in control rats at 52 weeks. The glomeruli and vasculature of control rats were not significantly labelled. (c) Nearly identical labelling

pattern of the renal tubules was observed in diabetic rats at 52 weeks. (d) $i$ NOS positive cells were rarely seen in control rat glomeruli at 52 weeks. (e, f) In contrast to the control rats, diabetic rats at 52 weeks displayed a strong hybridization signal in the podocytes (e, arrow) and glomerular macrophages (f, arrow). Magnification: a, b $\times 100, \mathbf{c} \times 400, \mathbf{d}-\mathbf{f} \times 1,000$ 


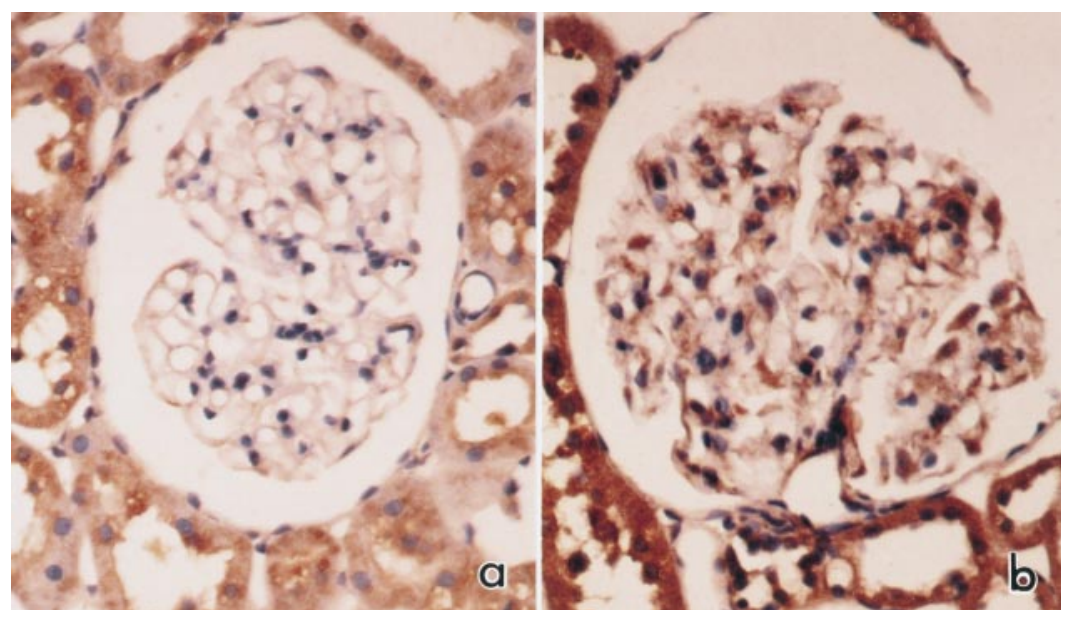

Fig. 7.a,b Immunohistochemical localization of TNF- $\alpha$ in (a) a control rat glomerulus at 52 weeks, (b) a STZ-induced diabetic rat glomerulus at 52 weeks

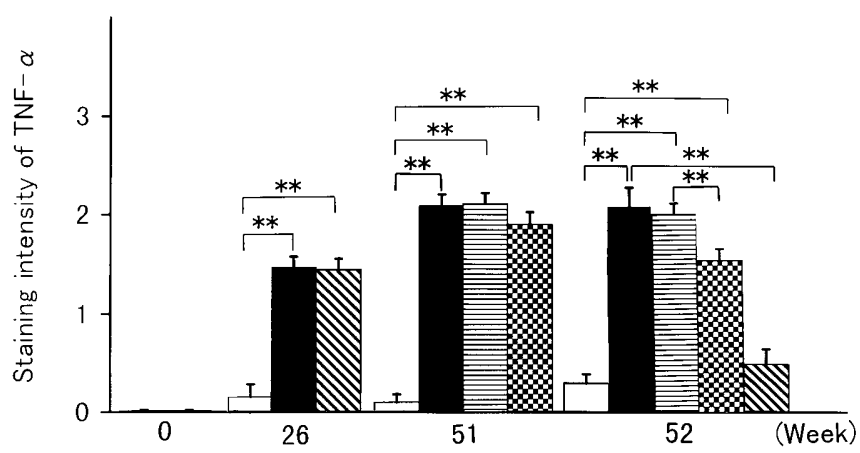

Fig. 8. Expression of TNF- $\alpha$ in STZ-induced diabetic rat glomeruli. The staining intensity of TNF- $\alpha$ in glomeruli of STZ-induced diabetic rats was increased compared with that in control rats at 52 weeks. Aminoguanidine therapy ameliorated its staining intensity. $* * p<0.01 ; \square \mathrm{CONT}, \square \mathrm{STZ}, \mathbb{N S T Z}+$ $\mathrm{AG}, \mathrm{STZ}+$ rabbit IgG, STZ + Anti-TNF

phorase was higher than in the control animals, thus confirming the results of the immunohistochemical studies for $i$ NOS (Fig. 5).

Expression of iNOS mRNA in the kidney. In control experiments, hybridization with the sense probe did not show any notable signals both in the normal and STZ-induced diabetic rats (Fig. 6a). The hybridization of kidney sections of the normal rats with the antisense $i$ NOS probe yielded positive signals of cortical thick ascending limb (TAL), proximal tubules and collecting ducts (Fig. 6b). The glomeruli and vasculature of control rats showed no signals (Fig. 6b). Similar $i$ NOS mRNA expression was detected in the renal tubules of diabetic rats. Diabetic rats at 52 weeks displayed, however, strong hybridization signals in the podocytes (Fig.6e, arrow) and glomerular macrophages compared with the control rats (Fig. 6f, arrow).
Staining for $T N F$ - $\alpha$. In control rats from 0 to 52 weeks, there was almost no staining for TNF- $\alpha$ in the glomeruli (Figs. $7 \mathrm{a}, 8$ ). The increased expression of TNF- $\alpha$ in the glomeruli of the diabetic group was noted at 52 weeks (Figs. 7b, 8). Treatment with aminoguanidine decreased the staining intensity of TNF- $\alpha$.

Urinary $\mathrm{NO}_{2} / \mathrm{NO}_{3}$ and glomerular $\mathrm{NO}_{2} / \mathrm{NO}_{3}$ production. From 26 to 52 weeks, in STZ-induced diabetic rats the urinary $\mathrm{NO}_{2} / \mathrm{NO}_{3}$ excretion showed no significant difference from the control animals (Fig.9). At 52 weeks diabetic rats treated with aminoguanidine showed decreased urinary $\mathrm{NO}_{2} / \mathrm{NO}_{3}$ excretion compared with control and diabetic rats. Although intraglomerular $i$ NOS expression was increased at 52 weeks in the diabetic group, urinary $\mathrm{NO}_{2} / \mathrm{NO}_{3}$ excretion showed no significant differences from the control animals. Therefore, we measured the glomerular $\mathrm{NO}_{2} / \mathrm{NO}_{3}$ production (Fig. 10).

Diabetic rats had increased glomerular $\mathrm{NO}_{2} / \mathrm{NO}_{3}$ excretion at 52 weeks compared with control rats (Fig.10). Aminoguanidine therapy prevented an increase in $\mathrm{NO}_{2} / \mathrm{NO}_{3}$ in diabetic rats and decreased nitrite concentrations to lower concentrations than those found in control rat glomeruli.

\section{Discussion}

Our study shows that intraglomerular AGE (CML) accumulation precedes the increase in the expression of TNF- $\alpha$ and $i \mathrm{NOS}$ and the production of $\mathrm{NO}_{2} / \mathrm{NO}_{3}$ in diabetic rat glomeruli. Treatment with aminoguanidine, which prevents AGE formation, reduced the glomerular expression of TNF- $\alpha$ and $i$ NOS, and glomerular $\mathrm{NO}_{2} / \mathrm{NO}_{3}$ production. These data suggest that overexpression of intraglomerular $i$ NOS could be caused by a CML-induced cytokine, i.e. TNF- $\alpha$, in diabetic glomeruli (CML-cytokine-NO sequence pathway). Moreover, intraglomerular expression of $i$ NOS paralleled glomerular $\mathrm{NO}_{2} / \mathrm{NO}_{3}$ production 


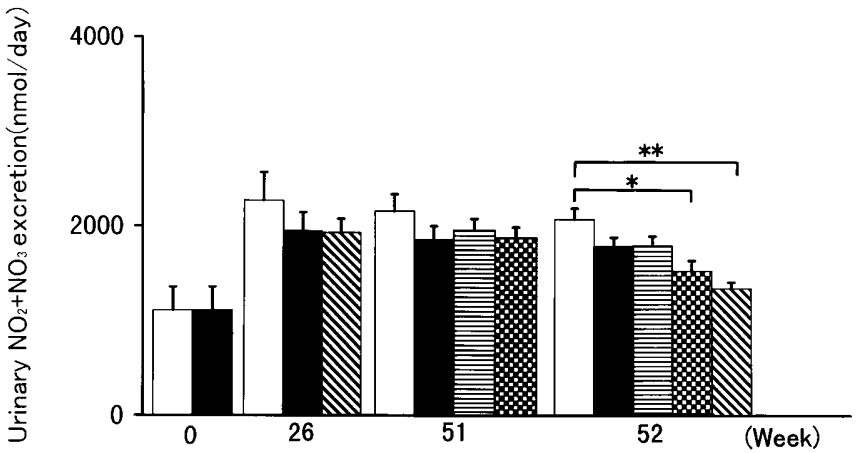

Fig.9. From 0 to 52 weeks, in STZ-induced diabetic rats the urinary $\mathrm{NO}_{2} / \mathrm{NO}_{3}$ excretion showed no significant difference from the control animals. At 52 weeks, diabetic rats treated with aminoguanidine showed decreased urinary $\mathrm{NO}_{2} / \mathrm{NO}_{3} \mathrm{ex}-$ cretion compared with control and diabetic rats. ${ }^{*} p<0.05$, $* * p<0.01 ; \square$ CONT, $\mathrm{STZ}, \mathbb{N S T Z}+\mathrm{AG}$, 囬STZ + rabbit $\mathrm{IgG}, \mathrm{STZ}+$ Anti-TNF

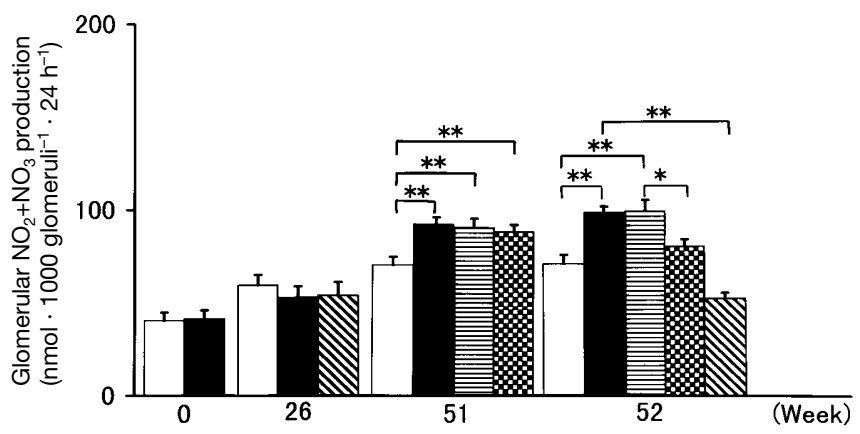

Fig. 10. Diabetic rats had increased glomerular $\mathrm{NO}_{2} / \mathrm{NO}_{3}$ concentrations at 52 weeks compared with control rats. Treatment with aminoguanidine or anti-TNF- $\alpha$ antibody prevented the increase in $\mathrm{NO}_{2} / \mathrm{NO}_{3}$ concentrations in diabetic rats and in aminoguanidine-treated diabetic rats decreased these concentrations to even less than that in control rat glomeruli. ${ }^{*} p<0.05$, $* * p<0.01 ; \square$ CONT, $\square$ STZ, $\mathbb{S T Z}+\mathrm{AG}$, 国 STZ + rabbit $\mathrm{IgG}, \mathrm{STZ}+$ Anti-TNF

and urinary protein excretion suggesting that overproduction of NO contributes to the development of diabetic nephropathy. Recently, it has been shown that AGE receptors are present on both macrophages and mesangial cells and that certain cytokines, such as TNF- $\alpha$ and interleukin- $1 \alpha$ (IL- $1 \alpha$ ) are produced after monocytic uptake and breakdown of AGEs. Treatment of mesangial cells with IL-1 or TNF- $\alpha$ has been shown to induce $i$ NOS [13]. It has been reported that AGE-proteins are possibly involved in the production of TNF- $\alpha$ and IL-1 from macrophages in diabetic nephropathy [14]. These data support our findings in vivo.

Receptors for AGEs provide the cell surface binding sites for AGEs. These receptors are localized on macrophages and mesangial cells [7, 8]. Many macrophages are found in the glomeruli of streptozotocininduced diabetic rats [15]. These findings suggest that the AGEs observed in the cells of our study may be explained by those phagocytosed by macrophages in the mesangial regions.

At 52 weeks, diabetic rats had more $i$ NOS-positive cells in their glomeruli and increased nitrite/nitrate production in glomerular supernatant after 24-h incubation than control rats. In the rats with STZ-induced diabetes, staining intensity of NADPH diaphorase was higher than in the control animals, thus supporting the results of the immunohistochemical studies for $i$ NOS. Glomerular AGE staining and the number of $i$ NOS-positive cells correlated with proteinuria. Increased urinary nitrite/nitrate excretion has recently been reported in diabetic rats even though no changes in $i$ NOS protein expression were detected [16]. This difference can possibly be explained by the duration of diabetes in the rats examined. Since an increase of $i$ NOS-positive cells was observed at 52 weeks and not at 26 weeks in our study, the observation period in that study ( 32 weeks) might have been insufficient to detect the increased expression of $i$ NOS.

Nitric oxide has been implicated in the inhibition of mitochondrial respiration, specifically by knocking out iron containing enzymes such as aconitase, NADH-ubiquinone and succinate-ubiquinone oxidoreductase [17]. Inhibition of glycolysis in parallel with inhibition of the respiratory chain and citric acid cycle will severely impair the cellular energy supply and contribute to the cytotoxic action of NO.

Our study shows that CML may enhance the expression of $i$ NOS by stimulating the expression of TNF- $\alpha$ in diabetic rat glomeruli. The CML-cytokineNO sequence pathway could be one of the major mechanisms in the development of diabetic nephropathy.

Acknowledgements. This work was supported by a grant-in-aid for scientific research from the Ministry of Education, Science, and Culture, Japan.

\section{References}

1. The Diabetes Control and Complications Trial Data Group (1993) The effect of intensive treatment of diabetes on the development and progression of long-term complications in insulin dependent diabetes mellitus. N Eng J Med 329: 977-986

2. Makino H, Shikata K, Hironaka K et al. (1995) Ultrastructure of nonenzymatically glycated mesangial matrix in diabetic nephropathy. Kidney Int 48: 517-526

3. Shikata K, Makino H, Sugimoto H et al. (1995) Localization of advanced glycation endproducts in the kidney of experimental diabetic rats. J Diabetes Complications 9: 269-271

4. Moncada S, Palmer RMJ, Higgs EA (1991) Nitric oxide: physiology, pathophysiology, and pharmacology. Pharmacol Rev 43: 109-142

5. Hibbs JB Jr, Taintor RR, Vavrin Z, Rachlin EM (1988) Nitric oxide: a cytotoxic activated macrophage effector molecule. Biochem Biophys Res Commun 157: 87-94 
6. Pfeilschifter J, Rob P, Mulsch A, Fandrey J, Vosbeck K, Busse R (1992) Interleukin-1- $\beta$ and tumor necrosis factor$\alpha$ induce a macrophage-type of nitric oxide synthase in rat renal mesangial cells. Eur J Biochem 203: 251-255

7. Skolnik EN, Yang Z, Makita Z, Radoff S, Kirstein M, Vlassara H (1990) Human and rat mesangial receptors glucosemodified proteins: potential role in kidney tissue remodeling and diabetic nephropathy J Exp Med 174: 631-939

8. Brett J, Schmidt AM, Dan SD et al. (1993) Survey of the distribution of a newly characterized receptor for advanced glycation end products in tissues. Am J Pathol 143: 1699-1712

9. Sugimoto H, Shikata K, Makino H, Ota K, Ota Z (1996) Increased gene expression of insulin-like growth factor-1 receptor in experimental diabetic rat glomeruli. Nephron 72: 648-653

10. Sugimoto H, Shikata K, Matsuda H, Oka K, Oka Z (1998) Increased expression of endothelial cell nitric oxide synthase (ecNOS) in afferent and glomerular endothelial cells is involved in glomerular hyperfiltration of diabetic nephropathy. Diabetologia 41: 1426-1434

11. Horiuchi S, Araki N, Morino Y (1991) Immunochemical approach to characterize advanced glycation end products of Maillard reaction. J Biol Chem 66: 7329-7332
12. Miyatake N, Shikata K, Sugimoto H et al. (1998) Intercellular adhesion molecule-1 mediates mononuclear cell infiltration into rat glomeruli after renal ablation. Nephron 79: 91-98

13. Pfeilschifter J, Vosbeck K (1991) Transforming growth factor $\beta 2$ inhibits interleukin $1 \beta$ and tumor necrosis factor $\alpha$ induction of nitric oxide synthase in rat renal mesangial cells. Biochem Biophys Res Comm 175: 372-379

14. Hasegawa G, Nakano K, Sawada M et al. (1991) Possible role of tumor necrosis factor and interleukin-1 in the development of diabetic nephropathy. Kidney Int 40: 1007-1012

15. Sugimoto H, Shikata K Hirata K et al. (1997) Increased expression of intercellular adhesion molecule-1 (ICAM-1) in diabetic rat glomeruli-glomerular hyperfiltration is a potential mechanism of ICAM-1 upregulation. Diabetes 46: 2075-2081

16. Soulis T, Cooper ME, Sastra S et al. (1997) Relative contributions of advanced glycation and nitric oxide synthase inhibition to aminoguanidine-mediated renoprotection in diabetic rats. Diabetologia 40: 1141-1151

17. Nathan C, Hibbs JB Jr (1991) Role of nitric oxide synthesis in macrophage antimicrobial activity. Curr Opin Immunol 3: $65-70$ 